\title{
Communication
}

\section{Reconstruction of Large Osteochondral Lesions in the Knee: Focus on Fixation Techniques}

\author{
Christian D. Weber ${ }^{1, *(\mathbb{D})}$, Filippo Migliorini ${ }^{2}$ and Frank Hildebrand $^{1}$ \\ 1 Department of Trauma and Reconstructive Surgery, RWTH Aachen University, Pauwelsstr. 30, \\ 52074 Aachen, Germany; fhildebrand@ukaachen.de \\ 2 Department of Orthopaedics and Trauma Surgery, RWTH Aachen University, 52074 Aachen, Germany; \\ migliorini.md@gmail.com \\ * Correspondence: chrweber@ukaachen.de; Tel.: +49-241-8036161
}

check for updates

Citation: Weber, C.D.; Migliorini, F.; Hildebrand, F. Reconstruction of Large Osteochondral Lesions in the Knee: Focus on Fixation Techniques. Life 2021, 11, 543. https:/ / doi.org/ $10.3390 /$ life11060543

Academic Editor: William John Ribbans

Received: 8 March 2021

Accepted: 4 June 2021

Published: 10 June 2021

Publisher's Note: MDPI stays neutral with regard to jurisdictional claims in published maps and institutional affiliations.

Copyright: (c) 2021 by the authors. Licensee MDPI, Basel, Switzerland. This article is an open access article distributed under the terms and conditions of the Creative Commons Attribution (CC BY) license (https:/ / creativecommons.org/licenses/by/ $4.0 /)$.

\begin{abstract}
Large $\left(>3 \mathrm{~cm}^{2}\right)$, focal osteochondral lesions (OCL) may result in poor functional outcomes and early secondary osteoarthritis of the knee. The surgical management of these OCL remains challenging. The treatment strategy must be tailored to various aspects, including lesion-specific (e.g., size, location, chronicity), joint-specific (e.g., instability, limb alignment, meniscal status), and patientspecific factors (e.g., age, activity level, comorbidities). Simple chondroplasty and bone marrow stimulation (BMS) techniques should be reserved for smaller lesions, as they only realize midterm clinical benefits, related to inferior wear characteristics of the induced fibrocartilage (type I collagen). Therefore, much attention has been focused on surgical restoration with hyaline cartilage (type II collagen), based on chondrocyte transplantation and matrix-assisted autologous chondrocyte implantation (MACI). Limited graft availability, staged procedures (MACI), and high treatment costs are limitations of these techniques. However, acute traumatic OCL of the femoral condyles and patellofemoral joint may also be suitable for preservation by surgical fixation. Early detection of the fragment facilitates primary repair with internal fixation. The surgical repair of the articular surface may offer promising clinical and cost-effective benefits as a first-line therapy but remains under-investigated and potentially under-utilized. As a unique characteristic, the fixation technique allows the anatomic restoration of the hyaline articular surface with native cartilage and the repair of the subchondral bone. In this manuscript, we present a case series of large OCL around the knee that were preserved by surgical fixation. Furthermore, various implants and techniques reported for this procedure are reviewed.
\end{abstract}

Keywords: large osteochondral lesion; flake fracture; articular fracture; osteochondral defect; knee joint; reconstructive techniques

\section{Introduction}

Osteochondral lesions (OCL) most frequently occur around the knee joint. Large OCL of the knee affect young and active individuals especially and may occur either as an isolated injury or in association with a ligamentous injury [1-5]. The prevalence of osteochondral lesions in common knee injuries, e.g., anterior cruciate ligament (ACL) tears and patella dislocations, is significant [6-8].

Large, displaced OCL with an intact cartilaginous surface are considered as an indication for surgical treatment. In these cases, where the salvage of the large fragment by internal fixation seems to be feasible, the preservation of the native cartilage is the theoretically ideal, single-stage, cost-effective, first-line technique for the high-loading components of the knee. In very large osteochondral fractures, internal fixation may even be the only rational therapeutic approach or an instrument to downsize the lesion.

The major principles of intraarticular fractures are also valid for the treatment of large OCL. These include the reconstruction of articular congruity, achieving stable fixation, restoring joint stability and allowing early joint motion [9]. Unfortunately, a significant 
proportion of these injuries are not diagnosed in the acute setting. For this reason, the existing body of literature is focused on the management of osteochondral defects, based on repair tissue stimulation, transplantation or regeneration of cartilage [10-13]. In the past literature, no consensus regarding the optimal management of OCL around the knee with one specific technique has been established, but a failure rate of $17 \%$ was reported [14].

A recent study suggests that in large osteochondral fractures after patella dislocation, internal fixation improves mid- and long-term outcomes when compared to debridement [15]. Furthermore, salvage techniques have been successfully applied even in latediagnosed large fragments $[16,17]$ or in chronic lesions, when combined with autologous bone grafting [18].

In light of the current literature, the spectrum of indications for internal fixation techniques for fragment preservation is evolving. We present the case-based management of three patients with large osteochondral fractures of weight-bearing knee components and review the current literature on salvage fixation techniques for these complex knee injuries.

\section{Material and Methods}

\subsection{Study Selection}

The study was conducted according to the guidelines of the Declaration of Helsinki, and approved by the local Ethics Committee (EK 224/21). Formal ethical statements of the included primary research may also be applicable. The study selection was not blinded for author, affiliation or source. An independent evaluation of the screened and discussion of the screened articles were performed by two reviewers (C.D.W., F.H.). The selection algorithm is presented in Figure 1.

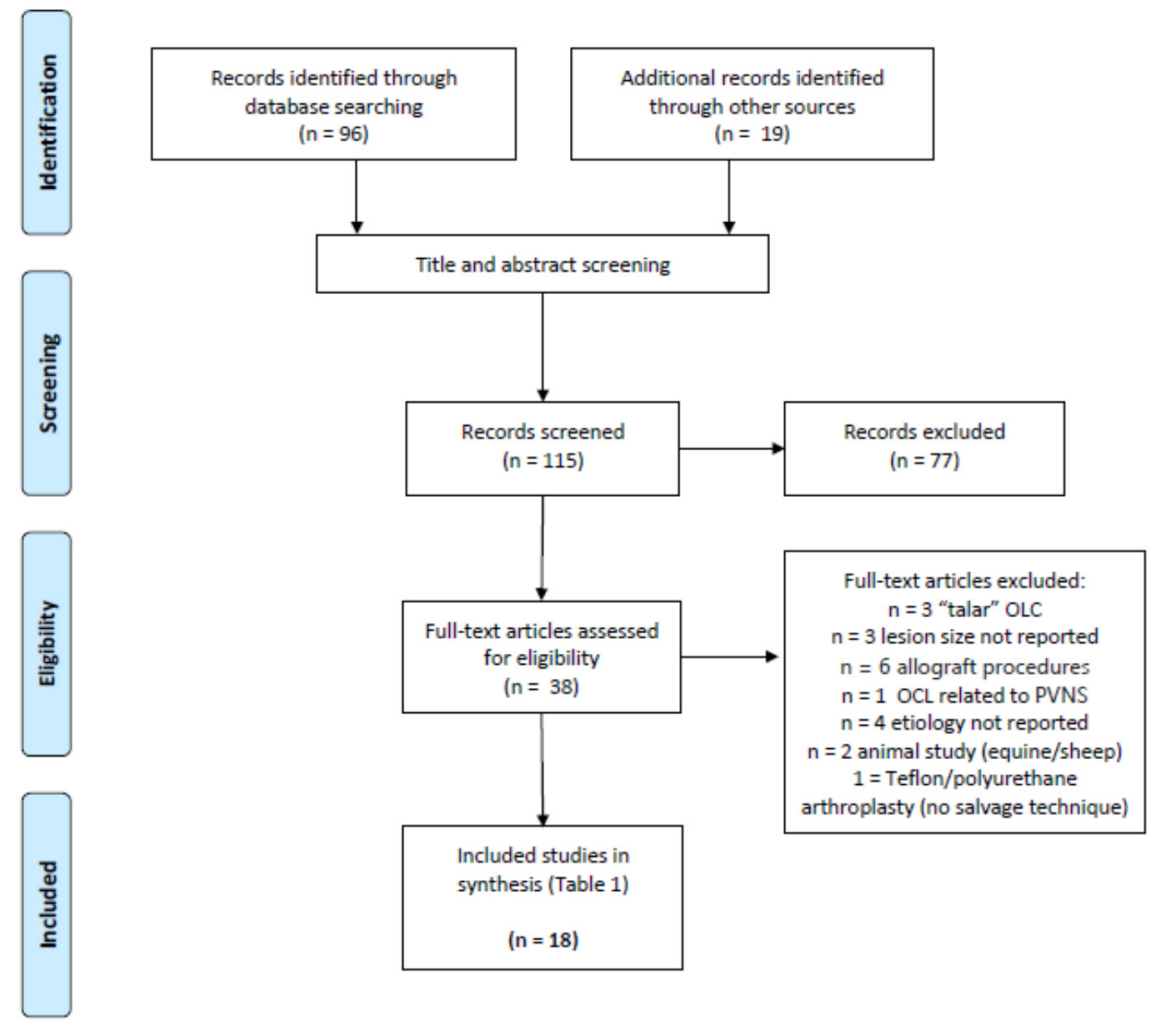

Figure 1. Study selection algorithm. Abbreviations: osteochondral lesion (OCL), pigmented villonodular synovitis (PVNS).

\subsection{Search Strategy}

The literature acquisition was based on electronic databases only (PubMed/Medline, Embase, and the Cochrane Library). Suitable clinical trials, experimental studies, as well 
as review articles published between 1 January 1990 and March 2021 were considered for inclusion. Animal studies were excluded. The following terms were used in the search strategy:

1. Anatomic region affected: "knee" OR "patella" OR "femoral condyle" OR "trochlea" NOT "elbow", OR “tibia", NOT "ankle", NOT "talus"

2. Entity/lesion type: AND "osteochondral lesion" OR "trans-chondral fracture" OR "osteo-/chondral" AND "lesion" OR "defect" OR "fracture" OR "flake" OR "injury" OR "fragment"

3. Surgical technique: AND "internal fixation" OR "repair" OR "fragment preservation" OR" salvage" OR "reconstruction"

4. Lesion size (optional, if characterized): "large" OR "massive"

Articles published in the English or German language were considered for selection. Additionally, a backward citation chaining strategy was applied.

\section{Case Presentation}

A 22-year-old male sustained a first-time patella dislocation in the left knee, and a large displaced fragment from the articular surface of the patella was identified radiographically (Figure 2a). A combined injury of the medial patellofemoral ligament (MPFL), medial patellotibial ligament (MPTL) and focal osteochondral lesions of the patellofemoral joint were confirmed in a subsequent magnetic resonance imaging (MRI; Figure 2b,c), and the large osteochondral fragment was located in the lateral joint space. The patellar fragment $(2.0 \times 2.0$ surface area, Figure 2d) was fixed after arthrotomy with three chondral darts (Figure 2e, Chondral Flap Repair System, Arthrex Inc., Naples, FL, USA) and additional monocryl sutures (Ethicon Inc., Somerville, NJ, USA). The MPFL was reconstructed with a quadriceps tendon graft technique (not shown). A small OCL of the lateral femoral condyle (LFC) was internally fixed with one chondral dart (Figure 2f,g). A partial-thickness chondral injury of the medial femoral condyle (MFC) was treated conservatively (Figure $2 \mathrm{~h}$ ). Radiographs confirmed the anatomic reconstruction of the patellar articular surface (Figure $2 \mathrm{i}$ ). After three months, a second-look arthroscopy confirmed the fragment stability with a small remaining defect (Figure $2 \mathrm{j}, \mathrm{k}$ ) covered with fibrocartilaginous tissue, while performing a revision for arthroscopic adhesiolysis.

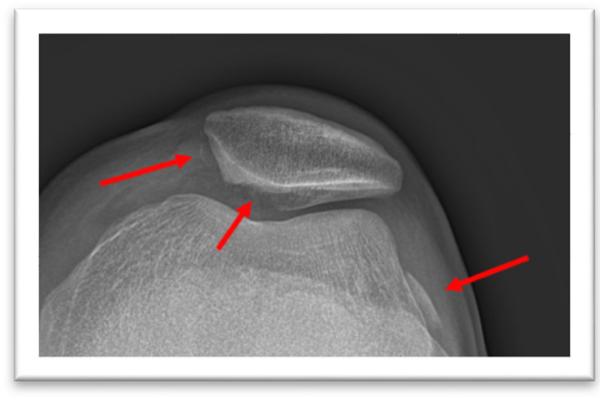

(a)

Figure 2. Cont. 


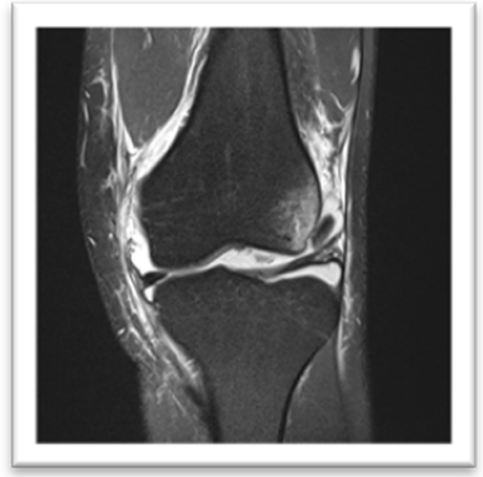

(b)

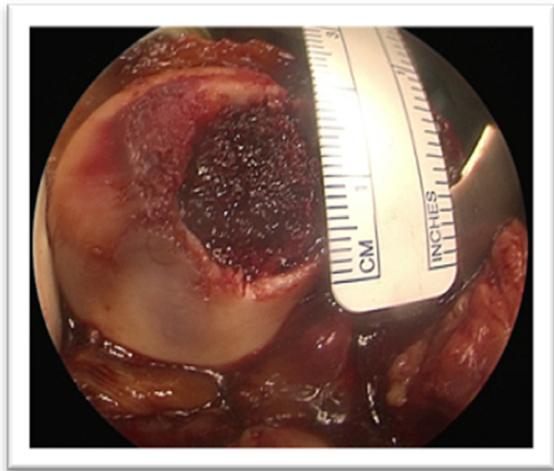

(d)

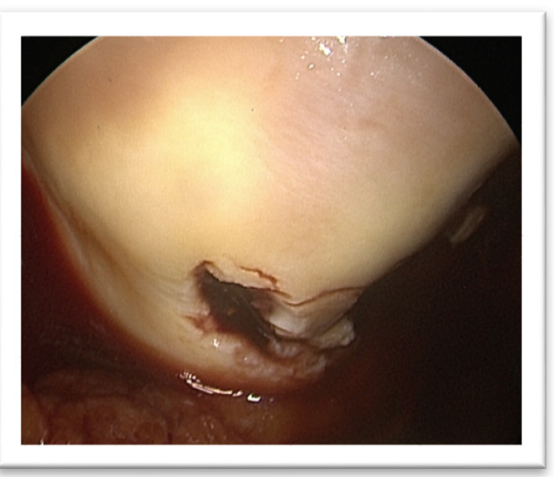

(f)

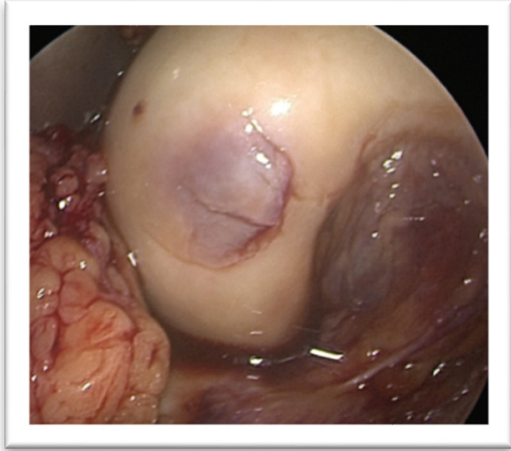

(h)

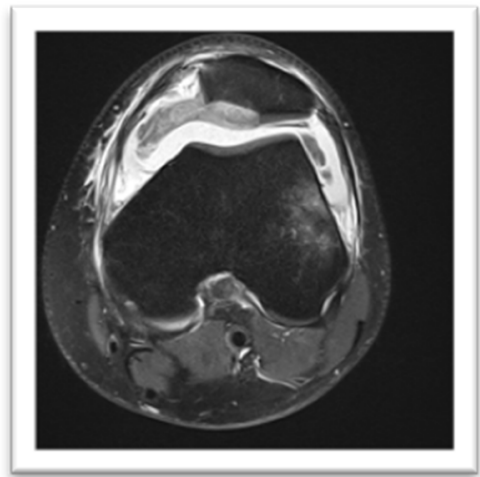

(c)

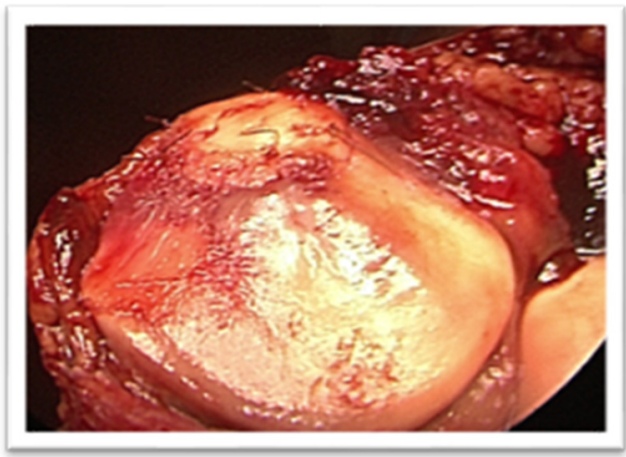

(e)

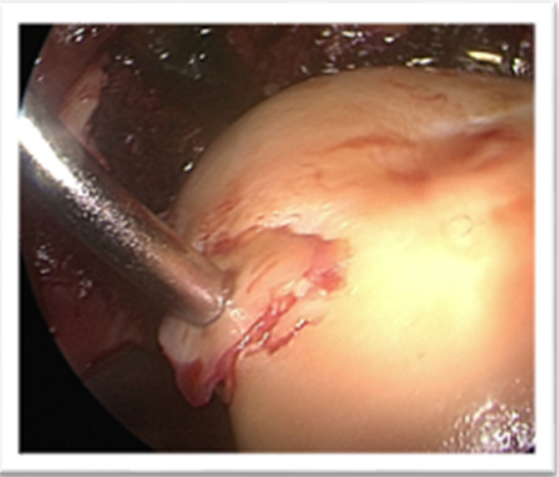

(g)

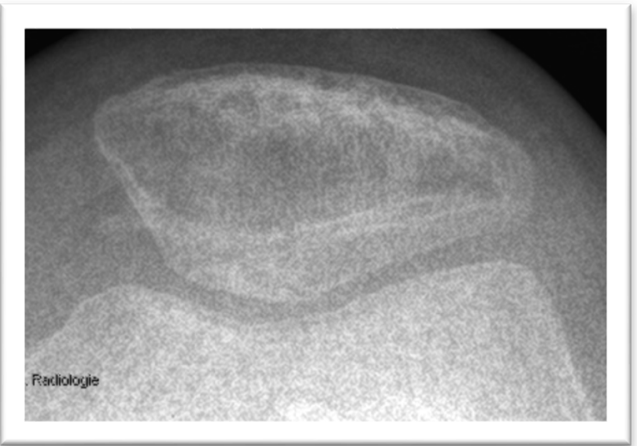

(i)

Figure 2. Cont. 


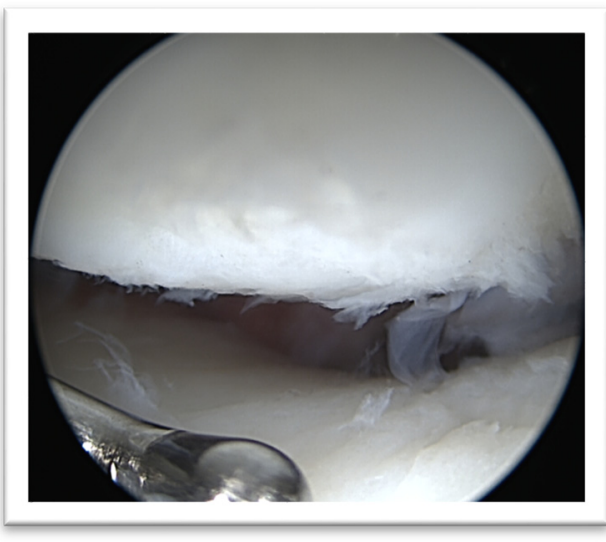

(j)

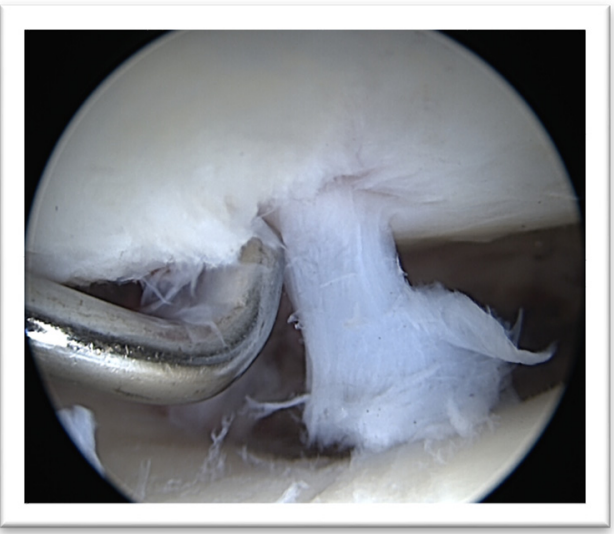

$(\mathbf{k})$

Figure 2. (a-i). Patella Dislocation. (a) Patella sunrise view, suggestive of MPFL and MPTL avulsion, a large osteochondral defect of the patella, and a displaced fragment in the lateral gutter. (b,c) MRI of the left knee, joint effusion, osteochondral fracture of the patella, displaced fragment in the lateral gutter, bone bruise of the lateral femoral condyle. (d-h) Intraoperative situation, osteochondral defect of the medial facet of the patella, before and after fragment fixation, small full-thickness osteochondral lesion of the lateral femoral condyle repaired with one chondral dart, partial-thickness chondral injury of the medial femoral condyle. (i) Postoperative patella sunrise view. (j,k). Arthroscopic re-evaluation three months postoperative: $(\mathbf{j})$ the osteochondral lesion is healed and stable under probing, (k) the small remaining defect is filled with fibrocartilaginous tissue.

\section{Case Presentation}

A 49-year-old male sustained a first-time patella dislocation while walking. A large osteochondral defect involving the entire weight-bearing aspect of the lateral condyle is visible, and the fragment is locking the patellofemoral joint (Figure 3a-e). Arthroscopicassisted internal fixation with absorbable darts was performed (Figure 3f-j; Chondral Flap Repair System, Arthrex Inc., Naples, FL, USA). The radiological follow-up shows the anatomic fragment position and healing of the subchondral bone three months post-injury, and the MRI after six months is presented (Figure $3 k-n$ ).

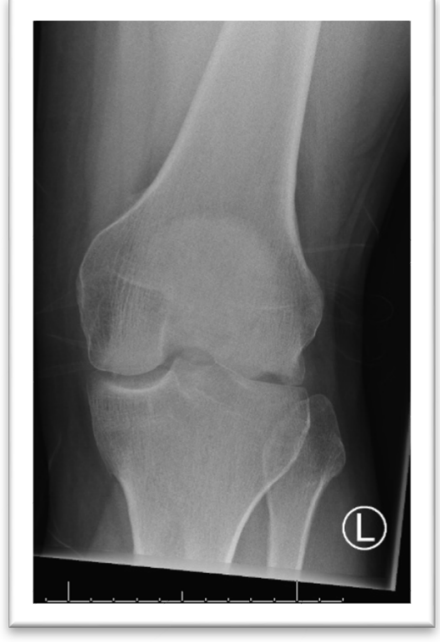

(a)

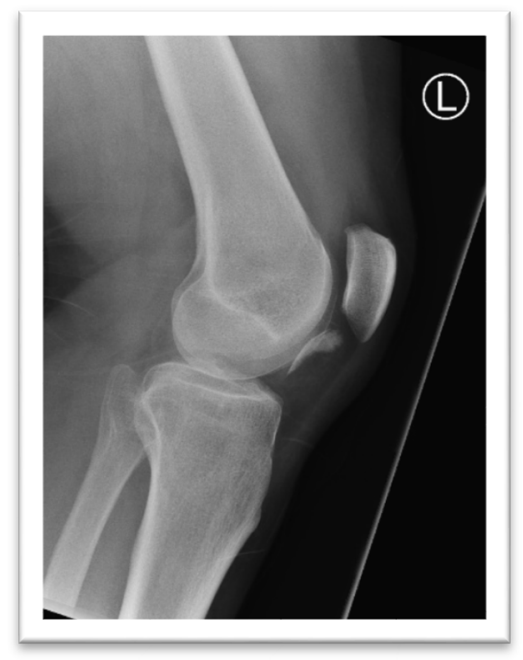

(b)

Figure 3. Cont. 


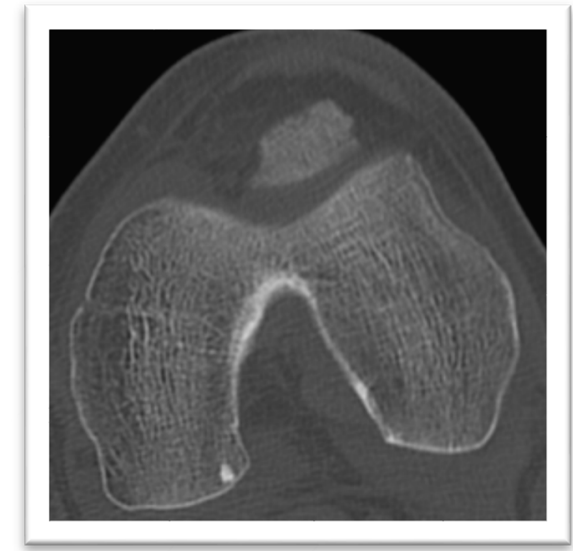

(c)

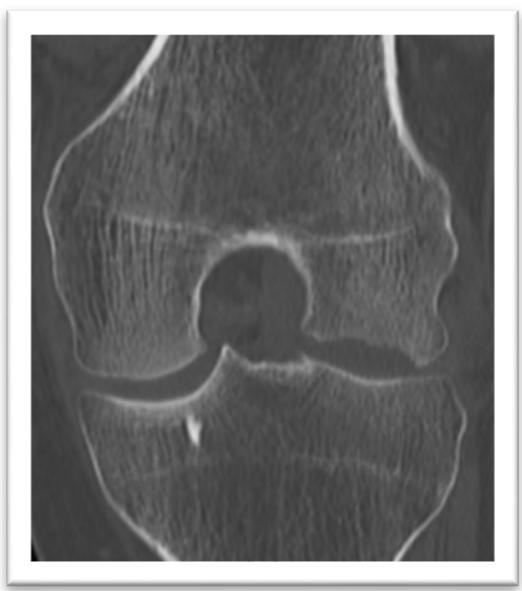

(e)

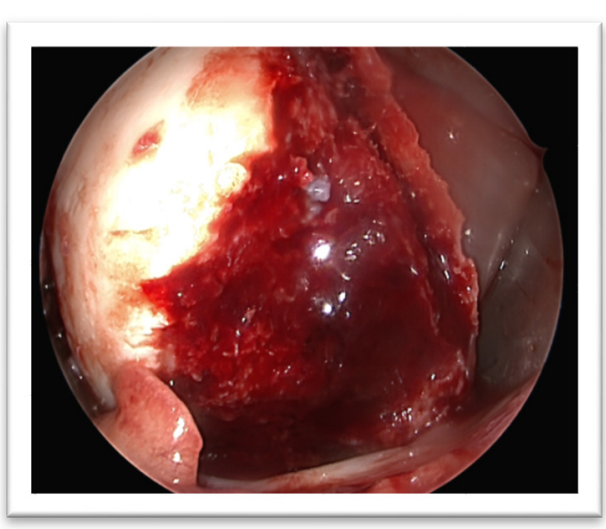

(g)

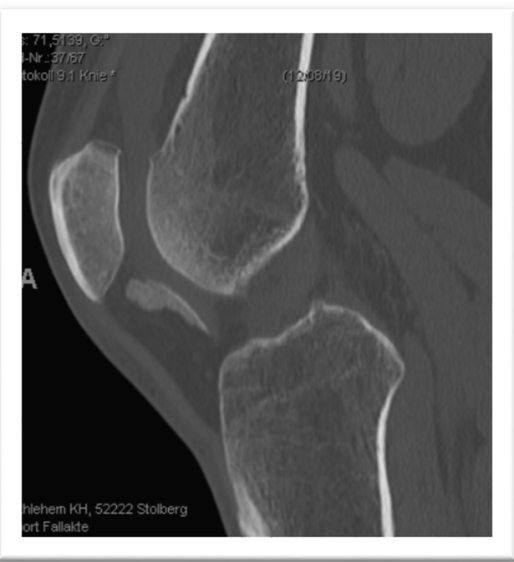

(d)

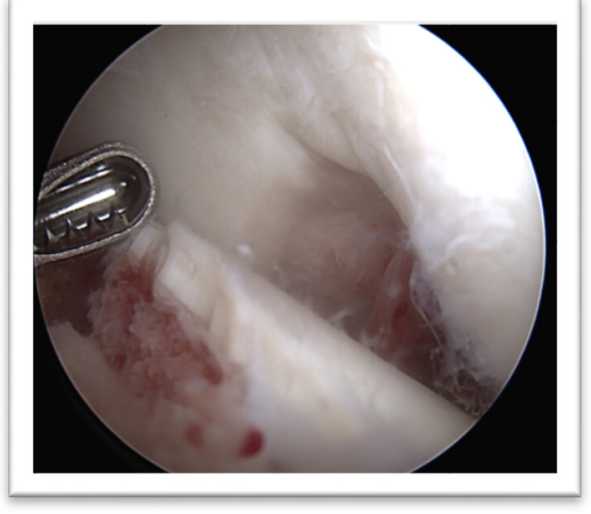

(f)

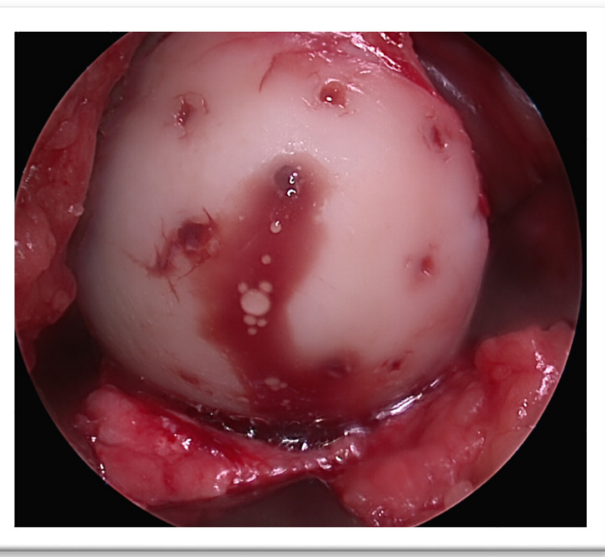

(h)

Figure 3. Cont. 


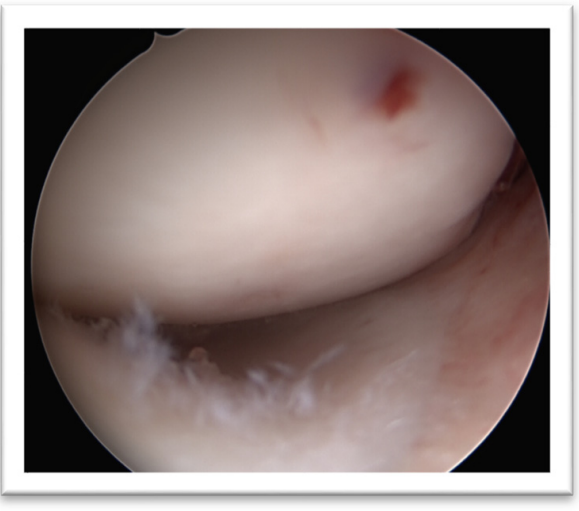

(i)

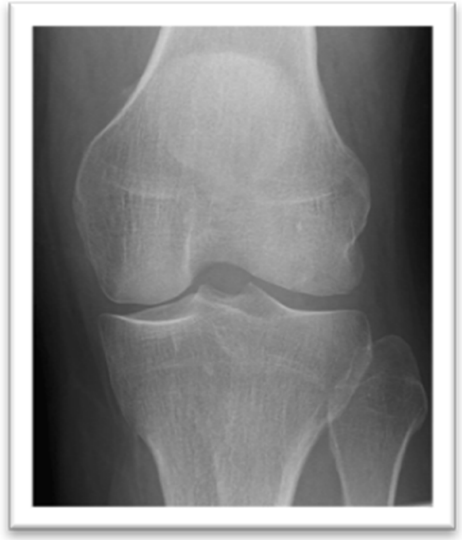

$(\mathbf{k})$

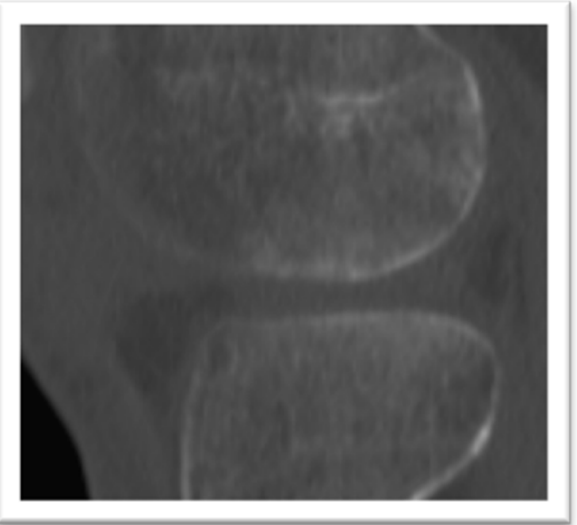

(m)

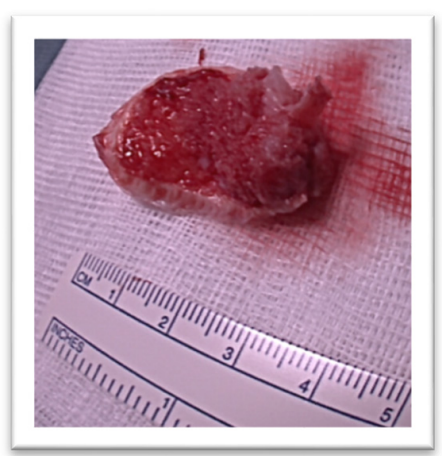

(j)

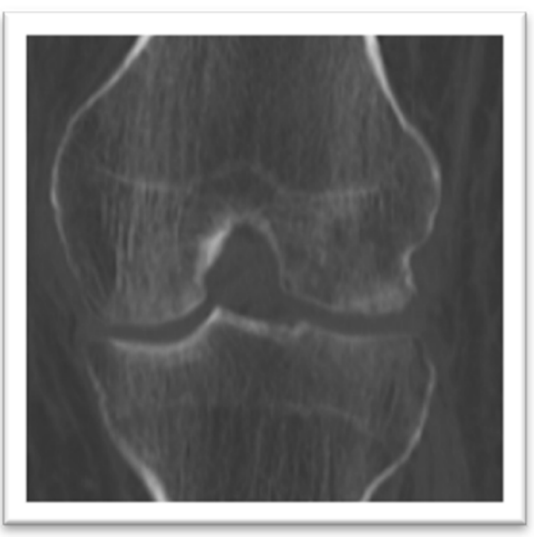

(1)

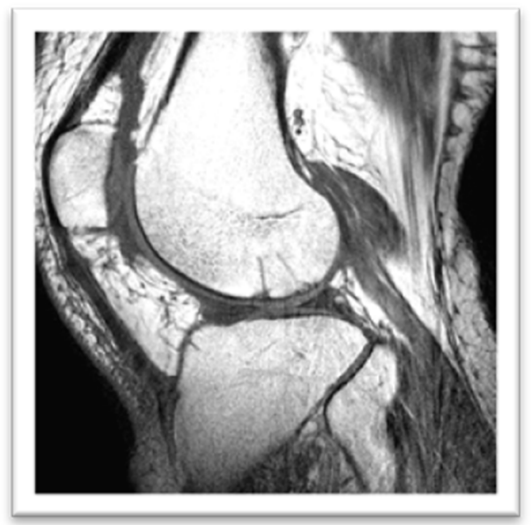

(n)

Figure 3. (a) Anterior-posterior radiograph of the left knee, limited range of motion due to the injury is obvious. (b) Lateral radiograph of the left knee, the osteochondral fragment is located inferior to the lower pole of the patella. (c-e) The coronal, axial and sagittal reconstruction during computed tomography (CT) reveals a massive osteochondral fracture of the lateral femoral condyle (LFC). (f) Arthroscopic evaluation of the displaced fragment. (g) The majority of the weight-bearing portion of the lateral femoral condyle is involved. (h) Reconstruction of the LFC after fixation with multiple PLLA darts. (i) Arthroscopic evaluation after internal fixation. (j) Osteochondral fragment with surface area measuring $3 \times 2.8 \mathrm{~cm}$, debridement was performed ex situ. (k) Postoperative AP radiograph shows anatomic articular alignment. $(\mathbf{l}, \mathbf{m})$ Sagittal and coronal CT reconstruction after three months shows adequate healing, but some subchondral sclerosis is present. (n) Sagittal MRI (T1) follow-up at six months, intact and vital cartilage surface, remaining small subchondral lesions at the sites of dart insertion. 


\section{Case Presentation}

A 34-year-old male sustained a high-energy motorcycle accident while the right knee was in a flexed position (Figure 4a,b). Axial and sagittal CT scans confirm the displaced injury with an articular step-off (Figure $4 \mathrm{c}, \mathrm{d}$ ). The majority of the lateral femoral condyle is fractured, attached only to the posterior capsule and partially to the ACL (Hoffa type, AO33-B3). The displaced fracture (Figure 4e,f) was anatomically reduced and fixed internally in a percutaneous anterior-posterior fashion (Figure $4 \mathrm{~g}$,h) under arthroscopic assistance (4.0 mm partially threaded, Fixos ${ }^{\circledR}$ headless compression screws, Stryker Inc., Kalamazoo, MI, USA). Proper visualization and identification of the ideal screw entry points preserving the femoral articular cartilage is facilitated under arthroscopic and fluoroscopic guidance (Figure $4 \mathrm{~h}$ ). The radiographic follow-up shows the anatomic position of the displaced fracture (Figure $4 \mathrm{i}, \mathrm{j}$ ) and adequate healing after three months via computed tomography (Figure 4k).

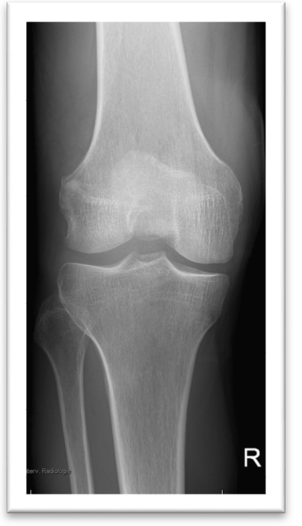

(a)

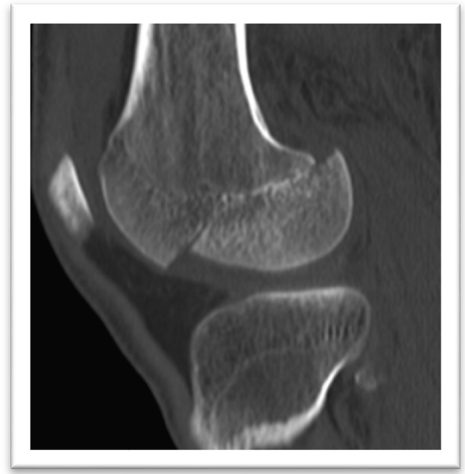

(c)

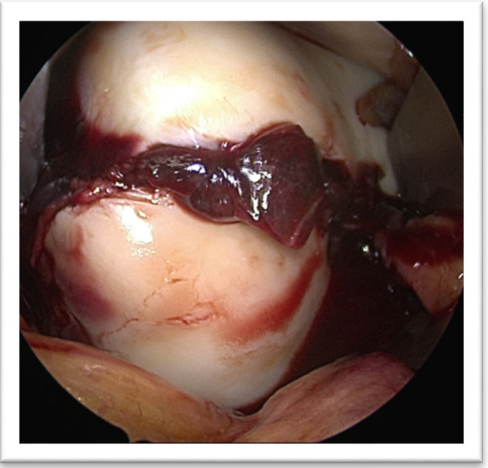

(e)

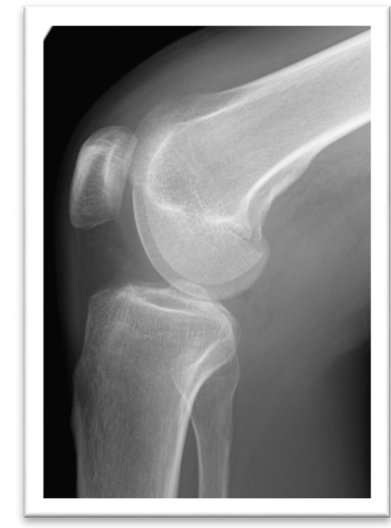

(b)

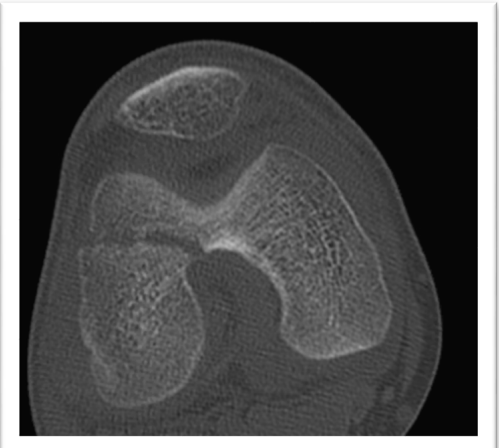

(d)

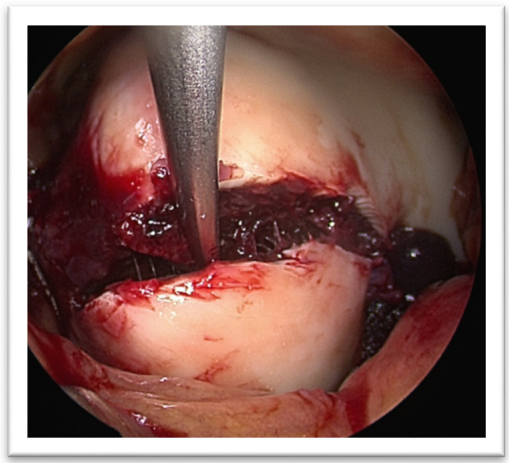

(f)

Figure 4. Cont. 


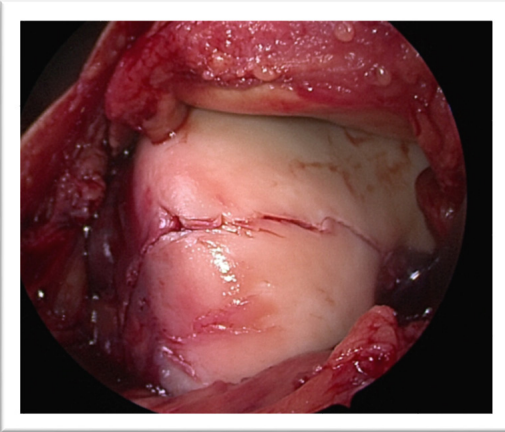

(g)

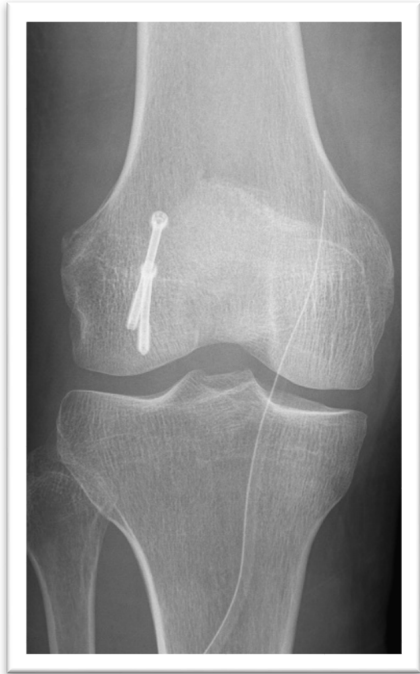

(i)

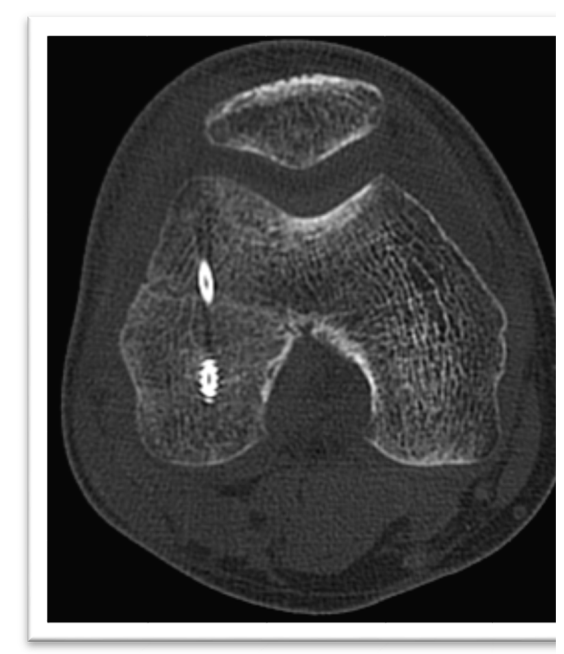

(k)

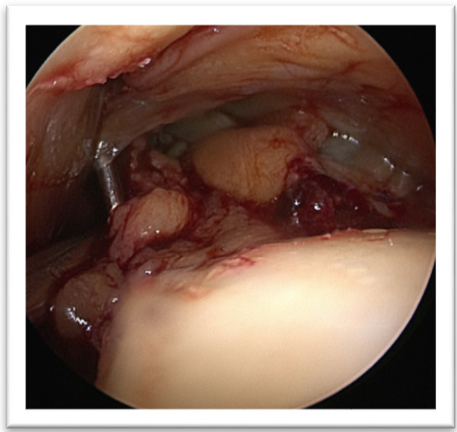

(h)

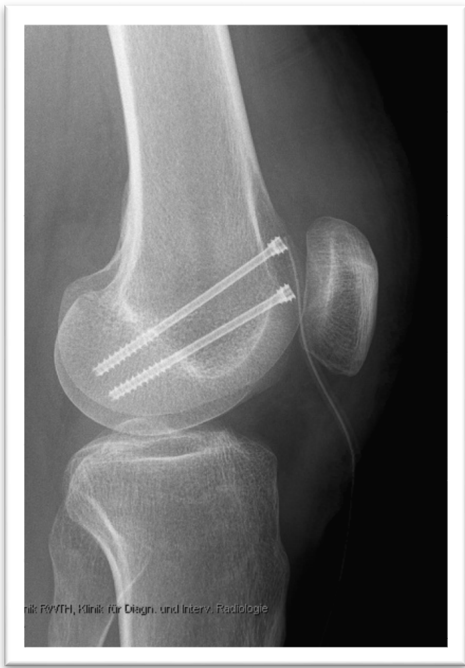

(j)

Figure 4. (a-k): (a) AP and (b) lateral radiograph of a Hoffa type fracture of the lateral femoral condyle, (c) sagittal and (d) axial assessment of the fracture morphology based on computed tomography (CT), (e) evaluation and (f) reduction for (g) anatomic reconstruction enables (h) arthroscopic assisted internal fixation; (i) AP and (j) lateral radiographic validation of screw positions and (k) follow-up CT. 


\section{Discussion}

Both direct and indirect trauma mechanisms may result in osteochondral lesions around the knee $[5,10]$. Anterior cruciate ligament (ACL) tears are associated with injuries to the lateral femoral condyle, and osteochondral lesions of the patellofemoral joint are common following patella dislocations $[19,20]$. In this context, a recent study evaluated the patterns of osteochondral fractures after acute or recurrent patella dislocation and reported that $63 \%$ affected the patella, $34 \%$ affected the lateral femoral condyle and only $3 \%$ affected both locations [21]. This distribution can be explained by anatomic characteristics, as the articular cartilage of the patella is softer when compared to the trochlea, is thicker when compared to any other joint in the human body, and does not follow the contour of the subchondral plate. Despite multiple anatomic variations of the patellofemoral joint, these aspects increase the likelihood of osteochondral shear injuries under high biomechanical loading. Osteochondral fractures of the patella may be larger after primary dislocation than after recurrent dislocation, most likely due to increased contact pressures. Accordingly, in the two cases presented with a patellofemoral pathology, both patients experienced a first-time dislocation of the patella and sustained a large osteochondral fracture of the patella and the lateral femoral condyle, respectively.

Song et al. evaluated an initial conservative treatment in sixty-nine patients with acute first-time patella dislocations. In the presence of large osteochondral fragments, the authors frequently observed a failure of the nonoperative treatment [22].

In the most recent treatment algorithms for patellar instability, recurrent patellofemoral instability or an osteochondral fracture are considered as an indication for surgical intervention $[23,24]$. In this context, Niemeyer et al. suggested that any osteochondral flake fracture indicates surgical treatment with the objective for internal fixation in both pediatric and adult patients [24]. Hence, due to the improved understanding of patellofemoral pathologies, a variety of arthroscopic and open surgical concepts for the repair of osteochondral lesions and the restoration of joint stability have been developed [25-29]. Furthermore, additional techniques for deformity correction (e.g., HTO) are inconsistently applied within the body of literature [30].

For patellofemoral pathologies, various chondral and osteochondral fixation techniques are suggested in combination with a wide spectrum of additional techniques, e.g., MPFL repair, MPFL reconstruction, medial reefing, lateral release. These methodological variances impede the scientific comparison of the reported study results. The reviewed literature predominantly comprises low level and quality evidence and is based on variable treatment protocols (Table 1). 
Table 1. Summary of study results after internal fixation of larger osteochondral and chondral lesions around the knee.

\begin{tabular}{|c|c|c|c|c|c|c|c|c|c|c|c|}
\hline Authors & Year & $\mathbf{N}$ & Age (y) & $\begin{array}{l}\text { Fragment } \\
\text { Composition }\end{array}$ & $\begin{array}{l}\text { Lesion Size/ } \\
\text { Surface Area }\end{array}$ & $\begin{array}{l}\text { Lesion } \\
\text { Chronicity }\end{array}$ & $\begin{array}{l}\text { Lesion } \\
\text { Location }\end{array}$ & $\begin{array}{l}\text { Fixation Device/ } \\
\text { Technique }\end{array}$ & $\begin{array}{l}\text { Additional } \\
\text { Procedures }\end{array}$ & $\begin{array}{l}\text { Complications/ } \\
\text { Reoperations }\end{array}$ & $\begin{array}{c}\text { Primary Outcomes } \\
\text { Evaluated at Follow-Up }\end{array}$ \\
\hline $\begin{array}{c}\text { Maletius and } \\
\text { Lundberg } \\
\text { [31] }\end{array}$ & 1994 & 2 & 18,21 & $\begin{array}{l}\text { Osteochondral } \\
\text { Chondral }\end{array}$ & $\begin{array}{c}2 \times 3 \mathrm{~cm} 2.5 \times \\
3.5 \mathrm{~cm}\end{array}$ & $\begin{array}{l}\text { Acute } \\
\text { Chronic }\end{array}$ & $\begin{array}{l}\text { Patella } \\
\text { LFC }\end{array}$ & $\begin{array}{l}\text { Fibrin sealant } \\
\text { and } \\
\text { polydioxanone } \\
\text { pins }\end{array}$ & $\begin{array}{l}1 \text { lateral } \\
\text { release }\end{array}$ & $\begin{array}{c}\text { 2nd look } \\
\text { arthroscopy }+ \\
\text { removal of } \\
\text { detached fragm. }\end{array}$ & $\begin{array}{l}\text { 7/8 mo: symptom free, } \\
\text { successful RTS }\end{array}$ \\
\hline $\begin{array}{l}\text { Dines et al. } \\
\text { [32] }\end{array}$ & 2008 & 9 & 18 & Osteochondral & $3.9 \mathrm{~cm}^{2}$ & $\begin{array}{l}1 \text { Acute } \\
8 \text { OCD }\end{array}$ & $\begin{array}{l}\text { Femoral } \\
\text { condyle }\end{array}$ & $\begin{array}{c}\text { self-reinforced } \\
\text { poly-L-lactic acid } \\
\text { device (PLLA) } \\
\text { nails }\end{array}$ & & $\begin{array}{l}\text { minimal } \\
\text { complication rate }\end{array}$ & $\begin{array}{l}\text { Lysholm score 94, } \\
\text { excellent } 7 \text {, good } 1 \text {, fair } 1\end{array}$ \\
\hline $\begin{array}{l}\text { Walsh et al. } \\
\text { [33] }\end{array}$ & 2008 & 8 & 13.1 & Osteochondral & $>4.0 \mathrm{~cm}^{2}$ & Acute & LFC (8) & $\begin{array}{l}\text { multiple } \\
\text { polyglycolic acid } \\
\text { rods }\end{array}$ & & $\begin{array}{c}4 \text { s look } \\
\text { arthroscopy, } \\
1 \text { unstable flap } \\
\text { removal (20\% of } \\
\text { surface area) }\end{array}$ & $\begin{array}{c}\text { Mean } \mathrm{f} / \mathrm{u} 9 \text { yr: } \\
\text { Cincinnati Knee rating: } \\
3 \text { excellent, } 2 \text { good, } 3 \text { fair, } \\
\text { no poor results }\end{array}$ \\
\hline $\begin{array}{l}\text { Chan et al. } \\
\text { [34] }\end{array}$ & 2014 & 1 & 12 & Chondral & $4 \times 2.2 \mathrm{~cm}$ & Acute & LFC & $\begin{array}{c}\text { 1.5-mm- } \\
\text { diameter } \\
\text { polylactide } \\
\text { fixation nails }\end{array}$ & MPFL repair & $\begin{array}{c}11.5 \text { mo staged } \\
\text { MPFL-R, healthy } \\
\text { cartilage observed }\end{array}$ & Uneventful healing \\
\hline $\begin{array}{l}\text { Barrett et al. } \\
\quad[35]\end{array}$ & 2016 & 22 & 21 & Osteochondral & $3.8 \mathrm{~cm}^{2}$ & OCD & $\begin{array}{l}\text { MFC (16) } \\
\operatorname{LFC~(6)~}\end{array}$ & $\begin{array}{l}\text { Metal headless, } \\
\text { cannulated } \\
\text { compression } \\
\text { screws }\end{array}$ & & $\begin{array}{l}4 \text { loose fragment } \\
\text { and hardware } \\
\text { removals: } 1 \text { wire } \\
\text { breakage, } 2 \text { screw } \\
\text { migrations }\end{array}$ & $\begin{array}{c}8.7 \mathrm{yr} \mathrm{f} / \mathrm{u}: 82 \% \text { union } \\
\text { rate, IKDC } 85 \\
\text { KOOS pain } 93 \\
\text { KOOS ADL } 98 \\
\text { KOOS Sports } 82\end{array}$ \\
\hline $\begin{array}{l}\text { Gesslein } \\
\text { et al. [15] }\end{array}$ & 2019 & 28 & 18.6 & Osteochondral & $3.7 \mathrm{~cm}^{2}$ & Acute & $\begin{array}{l}\text { Pat. (19) } \\
\text { LFC (9) }\end{array}$ & $1.3 \mathrm{~mm}$ BA pins & $\begin{array}{l}23 \text { medial } \\
\text { reefing }\end{array}$ & 2 reinterventions & $\begin{array}{c}7.9 \text { yr F/U, Lysholm 88.0, } \\
\text { IKDC 89.2, } \\
\text { KOOS Symptoms } 87.2\end{array}$ \\
\hline $\begin{array}{l}\text { Nuelle et al. } \\
\text { [36] }\end{array}$ & 2019 & 1 & NR & Osteochondral & $2.8 \times 2.5 \mathrm{~cm}$ & Acute & Patella & $\begin{array}{l}3 \text { mm PLLA } \\
\text { compression } \\
\text { screws }\end{array}$ & & NR & $\begin{array}{l}\text { Technical report only, } \\
\text { outcome NR }\end{array}$ \\
\hline $\begin{array}{l}\text { Churchill } \\
\text { et al. [37] }\end{array}$ & 2019 & 10 & 14.6 & Chondral & $3.86 \mathrm{~cm}^{2}$ & Acute & $\begin{array}{c}\text { Trochl.(4) } \\
\text { Patella (4) } \\
\text { LFC (2) }\end{array}$ & $\begin{array}{l}2.7 \mathrm{~mm} \text { BA or } \\
2.4 \mathrm{~mm} \text { metallic } \\
\text { compression } \\
\text { screws, } \\
\text { PLLA nails or } \\
\text { darts }\end{array}$ & $\begin{array}{l}2 \mathrm{MPFL} \\
\text { repair }\end{array}$ & $\begin{array}{c}3 \text { short-term } \\
\text { complications: } \\
2 \text { screw migrations, } \\
1 \text { BMS for failure } \\
\text { after } 1.34 \mathrm{y}\end{array}$ & $\begin{array}{c}56 \text { mo f/u: IKDC } 94.74 \\
\text { Marx 14.4, TAS } 7,90 \% \\
\text { RTS }\end{array}$ \\
\hline $\begin{array}{l}\text { Wang et al. } \\
{[38]}\end{array}$ & 2019 & 45 & $\begin{array}{l}14.9- \\
18.3\end{array}$ & Osteochondral & $2.7-3.8 \mathrm{~cm}^{2}$ & $28 \mathrm{OCD}$ & MFC (11) & $\begin{array}{l}\text { BA and metal } \\
\text { screws and/or } \\
\text { Kirschner wires }\end{array}$ & $\begin{array}{l}\text { ACLR 1, } \\
\text { OTA 2, } \\
\text { MMx 1 }\end{array}$ & $\begin{array}{c}13 \text { reoperations, } \\
2 \text { hardware } \\
\text { migrations, } 1 \text { late } \\
\text { BMS, } 8 \text { loose body } \\
\text { removals }\end{array}$ & $\begin{array}{c}\text { Kaplan-Meier } \\
\text { survivorship 1-year } \\
\text { 88.6\% and 5-year: } 68.8 \%\end{array}$ \\
\hline
\end{tabular}


Table 1. Cont.

\begin{tabular}{|c|c|c|c|c|c|c|c|c|c|c|c|}
\hline Authors & Year & $\mathbf{N}$ & Age (y) & $\begin{array}{c}\text { Fragment } \\
\text { Composition }\end{array}$ & $\begin{array}{l}\text { Lesion Size/ } \\
\text { Surface Area }\end{array}$ & $\begin{array}{l}\text { Lesion } \\
\text { Chronicity }\end{array}$ & $\begin{array}{l}\text { Lesion } \\
\text { Location }\end{array}$ & $\begin{array}{l}\text { Fixation Device/ } \\
\text { Technique }\end{array}$ & $\begin{array}{l}\text { Additional } \\
\text { Procedures }\end{array}$ & $\begin{array}{l}\text { Complications/ } \\
\text { Reoperations }\end{array}$ & $\begin{array}{c}\text { Primary Outcomes } \\
\text { Evaluated at Follow-Up }\end{array}$ \\
\hline $\begin{array}{l}\text { Schlechter } \\
\text { et al. [39] }\end{array}$ & 2019 & 38 & 14.7 & Osteochondral & $3.22 \mathrm{~cm}^{2}$ & $\begin{array}{l}17 \text { Acute } \\
15 \text { OCD }\end{array}$ & $\begin{array}{l}\text { Femur (23) } \\
\text { Patella (9) }\end{array}$ & $\begin{array}{l}3.0 \mathrm{~mm} \text { PLLA } \\
\text { compression } \\
\text { screws and/or } \\
1.3 \mathrm{~mm} \text { darts }\end{array}$ & $\begin{array}{l}\text { Staged } \\
\text { ACLR/MPF } \\
2 \text { MUA }\end{array}$ & $\begin{array}{c}6 \text { secondary proc., } \\
1 \text { for proud } \\
\text { implant, } \\
\text { none required } \\
\text { cartilage revision } \\
\text { proc. }\end{array}$ & $\begin{array}{c}59 \text { mo: Lysholm } 89.8 \\
\text { Pedi IKDC } 88.1 \text {, Tegner } \\
\text { postop. } 6.4\end{array}$ \\
\hline $\begin{array}{l}\text { Megremis } \\
\text { [40] }\end{array}$ & 2019 & 1 & 14 & Osteochondral & $>4 \mathrm{~cm}^{2}$ & Chronic & Trochlea & $1.5 \mathrm{~mm}$ BA pins & & & $\begin{array}{c}6 \text { mo: Healing, successful } \\
\text { RTS }\end{array}$ \\
\hline $\begin{array}{l}\text { Malecki et al. } \\
{[41]}\end{array}$ & 2019 & 17 & 14.1 & Osteochondral & $>1 \mathrm{~cm}^{2}$ & Acute & Patella & $\begin{array}{l}\text { Transpatellar } \\
\text { PDS suture } \\
\text { fixation }\end{array}$ & $\begin{array}{l}\text { MPFL rep. } \\
\text { if necessary }\end{array}$ & $\begin{array}{l}\text { Degenerative } \\
\text { changes in } \\
3 \text { patients }\end{array}$ & $\begin{array}{c}\text { Mean f/u } 7.5 \text { yr: } \\
\text { Lysholm 89.2, Kujala 89.6 }\end{array}$ \\
\hline $\begin{array}{l}\text { Jeuken et al. } \\
{[42]}\end{array}$ & 2019 & 3 & 12.8 & Chondral & $2,5,8 \mathrm{~cm}^{2}$ & $\begin{array}{c}\text { Acute } \\
1 \text { Chronic } 2\end{array}$ & $\begin{array}{l}\text { MFC (3), } \\
\text { Trochlea } \\
\quad(1)\end{array}$ & $\begin{array}{l}\text { Modified } \\
\text { Hedgehog } \\
\text { technique, fibrin } \\
\text { glue, suture }\end{array}$ & 1 ACLR & None at $1 \mathrm{yr} \mathrm{f} / \mathrm{u}$ & $\begin{array}{l}12 \text { mo: successful RTS, } \\
\text { full ROM, no pain, stable } \\
\text { fragment (MRI) }\end{array}$ \\
\hline $\begin{array}{l}\text { Beckert et al. } \\
{[43]}\end{array}$ & 2020 & 1 & 11 & Chondral & $2.2 \times 2 \mathrm{~cm}$ & Acute & LFC & $\begin{array}{c}3 \text { absorbable } \\
\text { nails }\end{array}$ & & $\begin{array}{l}\text { Healing confirmed } \\
\text { during arthroscopy }\end{array}$ & $\begin{array}{c}\text { RTS after } 6 \text { mo, elite level } \\
\text { at } 7 \text { mo }\end{array}$ \\
\hline $\begin{array}{c}\text { Ogura et al. } \\
{[44]}\end{array}$ & 2020 & 6 & 12.9 & Chondral & $3.8 \mathrm{~cm}^{2}$ & $\begin{array}{l}5 \text { Acute } \\
1 \text { OCD }\end{array}$ & $\begin{array}{l}\text { Trochlea } \\
\text { LFC }\end{array}$ & $\begin{array}{l}2.4 \mathrm{~mm} \\
\text { autologous bone } \\
\text { pegs }\end{array}$ & & $\begin{array}{c}1 \text { failure } 1.3 \text { yr after } \\
\text { a new injury }\end{array}$ & $\begin{array}{l}5.2 \mathrm{yr} F / \mathrm{U}, 85 \% \text { success } \\
\text { rate, RTS after } 7 \text { mo, } \\
\text { excellent KOOS in } 4\end{array}$ \\
\hline $\begin{array}{l}\text { Rüther et al. } \\
\quad[45]\end{array}$ & 2020 & 10 & 26.7 & Osteochondral & $3.33 \mathrm{~cm}^{2}$ & 10 Acute & $\begin{array}{l}\text { Femur (2) } \\
\text { Pat. (8) } \\
\text { Tibia (1) }\end{array}$ & $\begin{array}{l}\text { resorbable } \\
\text { polylactid } \\
\text { implants (screws, } \\
\text { nails, pins) }\end{array}$ & $\begin{array}{l}7 \text { med. } \\
\text { repair, lat. } \\
\text { release }\end{array}$ & NR & $\begin{array}{c}\text { 13.9 yr F/U: VASS 1.2, } \\
\text { Lysholm 85.7, } \\
\text { McDermott 90.7, Tegner } \\
4.4\end{array}$ \\
\hline $\begin{array}{c}\text { Zhou et al. } \\
\text { [46] }\end{array}$ & 2020 & 3 & 18 & Osteochondral & $1.5 \times 1.5 \mathrm{~cm}$ & Acute & LFC & $\begin{array}{l}\text { Titanium suture } \\
\text { anchor and } \\
\text { polydioxanone } \\
\text { suture (PDS) }\end{array}$ & & $\begin{array}{l}\text { 2nd-look } \\
\text { arthroscopy in all } \\
\text { patients }\end{array}$ & $\begin{array}{l}1 \mathrm{yr} \mathrm{F} / \mathrm{U} \text { : return to } \\
\text { physical activity and } \\
\text { uneventful healing }\end{array}$ \\
\hline $\begin{array}{l}\text { Gudemann } \\
\text { et al. [30] }\end{array}$ & 2021 & 15 & 17.7 & Chondral & $3.48 \mathrm{~cm}^{2}$ & $\begin{array}{l}3 \text { Acute } \\
12 \text { OCD }\end{array}$ & $\begin{array}{l}\text { MFC (8) } \\
\text { LCF (4) } \\
\text { Pat. (3) }\end{array}$ & $\begin{array}{c}\text { Screws (3), } \\
\text { PDS suture (9), } \\
\text { combination (3) }\end{array}$ & $1 \mathrm{HTO}$ & $\begin{array}{c}\text { 2nd-look } \\
\text { arthroscopy } \\
\text { showed loss of } \\
\text { fixation in } 3 \text { knees }\end{array}$ & $\begin{array}{c}80 \% \text { survival rate at } 4 \mathrm{yr} \\
\mathrm{F} / \mathrm{U}, \text { complete union in } \\
67 \% \text { (MRI) }\end{array}$ \\
\hline
\end{tabular}

Abbreviations: anterior cruciate ligament reconstruction (ACLR), Bone marrow stimulation (BMS), centimeter (cm), follow-up (F/U); high tibial osteotomy (HTO), International Knee

Documentation Committee (IKDC), Knee Injury and Osteoarthritis Outcome Score (KOOS), lateral femoral condyle (LFC), Marx Activity Rating Scale (Marx), manipulation under anesthesia (MUA), medial femoral condyle (MFC), medial meniscectomy (MMx), medial patellofemoral ligament (MPFL), month (mo), millimeter (mm), not reported (NR), osteochondrosis dissecans (OCD), polydioxanone sutures (PDS), poly-L-lactic acid (PLLA), range of motion (ROM), return to sports (RTS), year (yr). 
Furthermore, various methods of internal fixation are employed, including metallic (headless) and resorbable compression screws [23,24,26], Kirschner wires [26], resorbable polylactid implants (nails, pins and darts) [15,19-22,25,27,28,31,33], sutures (e.g., PDS) and suture-anchor constructs [29,34,35,47,48], fibrin sealants [19], and bone pegs [32]. Different main outcome measures and follow-up time periods are reported. To date, no consensus regarding a superior fixation technique or ideal characteristic for fragment preservation have been established.

Meanwhile, the expertise for smaller OCL, e.g., around the talus, is expanding. For the ankle, a consensus opinion has been achieved for several therapeutic aspects, including the fact that surgical fixation can be considered for acute and chronic lesions with intact fragments larger than $10 \mathrm{~mm}$ and $3 \mathrm{~mm}$ thick, but this is contraindicated in cases of generalized osteoarthritis [49]. In terms of the timing of the surgery, the fragment should be fixed as soon as possible to maximize the healing potential and to reduce the risk of secondary articular damage. These strategic considerations might theoretically apply for both the ankle and the knee joint.

As an example of a massive osteochondral fracture, the case of a Hoffa fracture has been illustrated (Case 3). This rare injury pattern consists of a very large osteochondral fracture of the femoral condyle in the coronal-plane and is most often related to highenergy trauma [50]. In general, vertical shear forces with varying degrees of knee flexion are responsible for this entity, which mandates surgical fixation [51]. Paradoxically, these large osteochondral fractures involving major portions of the femoral condyle (e.g., Hoffa fracture) may represent a diagnostic challenge because they may only be detected by the careful evaluation of the radiographs. In cases with inconclusive conventional radiographs, advanced imaging must be requested. Traditional surgical approaches are technically demanding and may involve a posterior buttress plate. The anatomic reduction of the articular surface and stable internal fixation are the principles of the surgical treatment. The arthroscopic-assisted reduction and minimally invasive internal fixation may improve outcomes, as an optimal screw placement can be validated and concomitant intraarticular injuries are identified arthroscopically [52,53].

These principles may also apply for large osteochondral lesions of the tibial plateau. However, the available literature on tibial lesions is sparse, and both osteochondral avulsion and impression fractures may occur at both the posterolateral (e.g., "apple-bite fracture") and posteromedial tibial plateaus $[54,55]$. Melugin et al. recently performed a review focusing on cartilage lesions of the tibial plateau [56]. The reported surgical techniques included osteochondral allograft and autograft transplantation, microfracture, osteochondral scaffolds and autologous chondrocyte transplantation, but no salvage procedures based on fixation techniques. The authors observed heterogeneous patient-reported outcomes and even deteriorated midterm outcomes after microfracture. In light of these results, the preservation of available osteochondral fragments requires further evaluation.

Major concerns in terms of fragment preservation remain in cases with chondral-only flaps and for those fragments which are only partially salvageable. Various authors reported a successful fixation and good outcomes even after the repair of chondral-only fragments, primarily in children and adolescents $[22,25,31,32,35]$. While some authors report that the subsequent swelling of the fragment is a concern in shear-off lesions because the anatomic reduction is impaired, a recent innovation takes advantage of the increased fragment dimensions. Jeuken et al. reported a modified Hedgehog technique in order to repair pure chondral shear-off lesions in pediatric knees [30]. Therefore, the chondral fragments were multiply incised and trimmed obliquely for an interlocking fit in the defect site, and the autograft was attached with fibrin glue and, if indicated, with sutures. However, the separation from the osteochondral unit is generally considered to be associated with a poor healing potential, especially in subacute cases and older patients.

A new concept for partial preservation has been suggested for partially salvageable fragments by the Mayo Clinic and Osaka University, Japan [57]. The group recently described a "hybrid technique" for the fixation of a partial fragment with absorbable 
compression screws or PLLA pins and an osteochondral autograft transplantation system (OATS) for the remaining defect. The authors reported positive outcomes and no complications in patients with a mean lesion size of $2.8 \mathrm{~cm} 2$ after a follow-up of 36 months.

In cases with a significant depression or fragmentation of large articular fragments, preservation may not be feasible at all [13]. For these cases, various techniques have been proposed, including (atelocollagen-associated) autologous chondrocyte implantation [58], the Mega-OATS technique [59], a combination of the osteochondral autograft transfer and the second-generation autologous chondrocyte implantation [60], as well as the minced cartilage implantation (MCI) procedure [61].

\section{Conclusions}

A high index of suspicion after acute knee injuries is vital for the early detection of large OCL, allowing the primary fixation of suitable fragments as a first-line therapy. Recent innovations including partial fragment preservation and "hybrid techniques" may increase the volume of salvaged hyaline cartilage and native subchondral bone. The wider application of arthroscopic techniques may be beneficial for the detection of concomitant pathologies, validation of anatomic fixation and improved outcomes. Compression and rotational fragment stability can be achieved by various surgical implants, and for some absorbable implants long-term outcomes are even available. For failed fixations, the management principles for osteochondral defects apply as second-line procedures. For specific treatment algorithms, a higher level and quality of evidence from future investigations is necessary.

Author Contributions: Conceptualization, C.D.W. and F.H.; Formal analysis, C.D.W., F.M. and F.H.; Methodology, C.D.W., F.M. and F.H.; Project administration, C.D.W. and F.H.; Resources, C.D.W. and F.H.; Validation, C.D.W. and F.H.; Visualization, C.D.W. and F.H.; Writing-original draft, C.D.W.; Writing-review and editing, F.M. and F.H. All authors have read and agreed to the published version of the manuscript.

Funding: This research received no external funding.

Institutional Review Board Statement: The study was conducted according to the guidelines of the Declaration of Helsinki, and approved by the local Ethics Committee (EK 224/21). Formal ethical statements of the included primary research may also be applicable.

Informed Consent Statement: Permission for the open access publication of patient images and details were approved including obtaining individual consent from all affected individuals.

Conflicts of Interest: The authors declare no conflict of interest with respect to the research, authorship, and/or publication of this article.

\section{References}

1. Krych, A.J.; Saris, D.B.F.; Stuart, M.J.; Hacken, B. Cartilage Injury in the Knee: Assessment and Treatment Options. J. Am. Acad Orthop. Surg. 2020, 28, 914-922. [CrossRef] [PubMed]

2. Valtanen, R.S.; Arshi, A.; Kelley, B.V.; Fabricant, P.D.; Jones, K.J. Articular Cartilage Repair of the Pediatric and Adolescent Knee with Regard to Minimal Clinically Important Difference: A Systematic Review. Cartilage 2020, 11, 9-18. [CrossRef] [PubMed]

3. Coughlin, R.P.; Gupta, A.; Sogbein, O.A.; Shanmugaraj, A.; Kurz, A.Z.; Simunovic, N.; Yen, Y.M.; Ayeni, O.R. Cartilage Restoration in the Adolescent Knee: A Systematic Review. Curr. Rev. Musculoskelet. Med. 2019, 12, 486-496. [CrossRef] [PubMed]

4. Gomoll, A.H.; Farr, J.; Gillogly, S.D.; Kercher, J.; Minas, T. Surgical management of articular cartilage defects of the knee. J. Bone Joint Surg. Am. 2010, 92, 2470-2490. [PubMed]

5. Ho, T.K.; Fang, D. Posterior cruciate avulsion fracture associated with a large inverted medial tibial osteochondral fragment. J. Trauma 1995, 38, 653-657. [CrossRef]

6. Chavez, A.; Jimenez, A.E.; Riepen, D.; Schell, B.; Khazzam, M.; Coyner, K.J. Anterior Cruciate Ligament Tears: The Impact of Increased Time From Injury to Surgery on Intra-articular Lesions. Orthop. J. Sports Med. 2020, 8, 2325967120967120. [CrossRef] [PubMed]

7. Maffulli, N.; Binfield, P.M.; King, J.B. Articular cartilage lesions in the symptomatic anterior cruciate ligament-deficient knee. Arthroscopy 2003, 19, 685-690. [CrossRef]

8. Nomura, E.; Inoue, M.; Kurimura, M. Chondral and osteochondral injuries associated with acute patellar dislocation. Arthroscopy 2003, 19, 717-721. [CrossRef] 
9. Mittal, S.; Mittal, R. Intra-articular Fractures: Principles of Fixation. In Intraarticular Fractures: Minimally Invasive Surgery, Arthroscopy; Doral, M.N., Karlsson, J., Nyland, J., Benedetto, K.P., Eds.; Springer International Publishing: Cham, Switzerland, 2019. [CrossRef]

10. Pedersen, M.E.; DaCambra, M.P.; Jibri, Z.; Dhillon, S.; Jen, H.; Jomha, N.M. Acute Osteochondral Fractures in the Lower Extremities-Approach to Identification and Treatment. Open Orthop. J. 2015, 9, 463-474. [CrossRef]

11. Chahla, J.; Hinckel, B.B.; Yanke, A.B.; Farr, J.; Bugbee, W.D.; Carey, J.L.; Cole, B.J.; Crawford, D.C.; Fleischli, J.E.; Getgood, A.; et al. An Expert Consensus Statement on the Management of Large Chondral and Osteochondral Defects in the Patellofemoral Joint. Orthop. J. Sports Med. 2020, 8, 2325967120907343. [CrossRef] [PubMed]

12. Calcei, J.G.; Ray, T.; Sherman, S.L.; Farr, J. Management of Large Focal Chondral and Osteochondral Defects in the Knee. J. Knee Surg. 2020, 33, 1187-1200. [CrossRef] [PubMed]

13. Seo, S.S.; Kim, C.W.; Jung, D.W. Management of focal chondral lesion in the knee joint. Knee Surg. Relat. Res. 2011, 23, 185-196. [CrossRef]

14. Kühle, J.; Angele, P.; Balcarek, P.; Eichinger, M.; Feucht, M.; Haasper, C.; Alexander, G.; Jung, T.; Lill, H.; Marquass, B.; et al. Treatment of osteochondral fractures of the knee: A meta-analysis of available scientific evidence. Int. Orthop. 2013, 37, 2385-2394. [CrossRef] [PubMed]

15. Gesslein, M.; Merkl, C.; Bail, H.J.; Krutsch, V.; Biber, R.; Schuster, P. Refixation of Large Osteochondral Fractures After Patella Dislocation Shows Better Mid- to Long-Term Outcome Compared with Debridement. Cartilage 2019. [CrossRef]

16. Enea, D.; Busilacchi, A.; Cecconi, S.; Gigante, A. Late-diagnosed large osteochondral fracture of the lateral femoral condyle in an adolescent: A case report. J. Pediatr. Orthop. B 2013, 22, 344-349. [CrossRef] [PubMed]

17. Hoshino, C.M.; Thomas, B.M. Late repair of an osteochondral fracture of the patella. Orthopedics 2010, 33. [CrossRef] [PubMed]

18. Herring, M.J.; Knudsen, M.L.; Macalena, J.A. Open Reduction, Bone Grafting, and Internal Fixation of Osteochondritis Dissecans Lesion of the Knee. JBJS Essent Surg. Tech. 2019, 9, e23. [CrossRef] [PubMed]

19. Rosslenbroich, S.; Raschke, M.; Petersen, W. Acute osteochondral lesions after patella dislocation. Unfallchirurg 2012, 115, 392-396. [CrossRef] [PubMed]

20. Lee, B.J.; Christino, M.A.; Daniels, A.H.; Hulstyn, M.J.; Eberson, C.P. Adolescent patellar osteochondral fracture following patellar dislocation. Knee Surg. Sports Traumatol. Arthrosc. 2013, 21, 1856-1861. [CrossRef] [PubMed]

21. Uimonen, M.; Ponkilainen, V.; Paloneva, J.; Mattila, V.M.; Nurmi, H.; Repo, J.P. Characteristics of Osteochondral Fractures Caused by Patellar Dislocation. Orthop. J. Sports Med. 2021, 9, 2325967120974649. [CrossRef]

22. Song, S.Y.; Kim, T.S.; Seo, Y.J. Initial conservative treatment of osteochondral fracture of the patella following first-time patellar dislocation. BMC Musculoskelet Disord. 2020, 21, 617. [CrossRef]

23. El Attal, R.; Zimmermann, F.; Balcarek, P. Instabile Patella des Erwachsenen. Arthroskopie 2020, 33, 420-427. [CrossRef]

24. Niemeyer, P.; Langenbrunner, S.; Schmal, H.; Salzmann, G.; Südkamp, N.P. Osteochondrosis dissecans und osteochondrale Verletzungen des Kniegelenks bei Kindern und Jugendlichen. Arthroskopie 2012, 25, 243-251. [CrossRef]

25. Bell, R.; Jimenez, A.E.; Levy, B.J.; Willson, R.; Arciero, R.A.; Edgar, C.M. Use of a Superolateral Portal and $70^{\circ}$ Arthroscope to Optimize Visualization of Patellofemoral Tracking and Osteochondral Lesions in Patients with Recurrent Patellar Instability. Arthrosc. Tech. 2020, 9, e1731-e1736. [CrossRef] [PubMed]

26. Migliorini, F.; Maffulli, N.; Eschweiler, J.; Quack, V.; Tingart, M.; Driessen, A. Lateral retinacular release combined with MPFL reconstruction for patellofemoral instability: A systematic review. Arch. Orthop. Trauma Surg. 2021, 141, 283-292. [CrossRef] [PubMed]

27. Maffulli, N.; Aicale, R.; D'Addona, A.; Young, D.A.; Kader, D.F.; Oliva, F. Combined medial patellofemoral and patellotibial reconstruction with soft tissue fixation in recurrent patellar dislocation. Injury 2020, 51, 1867-1873. [CrossRef] [PubMed]

28. Rath, B.; Eschweiler, J.; Betsch, M.; Gruber, G. [Cartilage repair of the knee joint]. Orthopade 2017, 46, 919-927. [CrossRef]

29. Richter, D.L.; Schenck, R.C., Jr.; Wascher, D.C.; Treme, G. Knee Articular Cartilage Repair and Restoration Techniques: A Review of the Literature. Sports Health 2016, 8, 153-160. [CrossRef] [PubMed]

30. Gudeman, A.; Wischmeier, D.; Farr, J. Surgical Fixation of Chondral-Only Fragments of the Knee: A Case Series with a Mean 4-Year Follow-up. Orthop. J. Sports Med. 2021, 9, 2325967120961391. [CrossRef] [PubMed]

31. Maletius, W.; Lundberg, M. Refixation of large chondral fragments on the weight-bearing area of the knee joint: A report of two cases. Arthroscopy 1994, 10, 630-633. [CrossRef]

32. Dines, J.S.; Fealy, S.; Potter, H.G.; Warren, R.F. Outcomes of osteochondral lesions of the knee repaired with a bioabsorbable device. Arthroscopy 2008, 24, 62-68. [CrossRef] [PubMed]

33. Walsh, S.J.; Boyle, M.J.; Morganti, V. Large osteochondral fractures of the lateral femoral condyle in the adolescent: Outcome of bioabsorbable pin fixation. J. Bone Joint Surg. Am. 2008, 90, 1473-1478. [CrossRef] [PubMed]

34. Chan, C.M.; King, J.J., 3rd; Farmer, K.W. Fixation of chondral fracture of the weight-bearing area of the lateral femoral condyle in an adolescent. Knee Surg. Sports Traumatol. Arthrosc. 2014, 22, 1284-1287. [CrossRef] [PubMed]

35. Barrett, I.; King, A.H.; Riester, S.; van Wijnen, A.; Levy, B.A.; Stuart, M.J.; Krych, A.J. Internal Fixation of Unstable Osteochondritis Dissecans in the Skeletally Mature Knee with Metal Screws. Cartilage 2016, 7, 157-162. [CrossRef] [PubMed] 
36. Nuelle, C.W.; Nuelle, J.A.V.; Balldin, B.C. Open Reduction Internal Fixation of a Traumatic Osteochondral Lesion of the Patella with Bioabsorbable Screw Fixation. Arthrosc. Tech. 2019, 8, e1361-e1365. [CrossRef] [PubMed]

37. Churchill, J.L.; Krych, A.J.; Lemos, M.J.; Redd, M.; Bonner, K.F. A Case Series of Successful Repair of Articular Cartilage Fragments in the Knee. Am. J. Sports Med. 2019, 47, 2589-2595. [CrossRef] [PubMed]

38. Wang, K.; Waterman, B.; Dean, R.; Redondo, M.; Cotter, E.; Manning, B.; Yanke, A.; Cole, B. The Influence of Physeal Status on Rate of Reoperation After Arthroscopic Screw Fixation for Symptomatic Osteochondritis Dissecans of the Knee. Arthroscopy 2020 36, 785-794. [CrossRef]

39. Schlechter, J.A.; Nguyen, S.V.; Fletcher, K.L. Utility of Bioabsorbable Fixation of Osteochondral Lesions in the Adolescent Knee: Outcomes Analysis with Minimum 2-Year Follow-up. Orthop. J. Sports Med. 2019, 7, 2325967119876896. [CrossRef] [PubMed]

40. Megremis, P.; Megremis, O.; Margariti, R. Late Repair, One Year After a Knee Twisting Injury, of a Missed Femoral Trochlea Osteochondral Fragment, With Bioabsorbable Nails, in a 14-Year-Old Boy. J. Am. Acad. Orthop. Surg. Glob. Res. Rev. 2019, 3, e040. [CrossRef] [PubMed]

41. Małecki, K.; Pruchnik-Witosławska, K.; Gwizdała, D.; Grzelak, P.; Flont, P.; Niedzielski, K.R. Clinical Results and MRI Evaluation of Patellar Osteochondral Fracture Fixation following Patellar Dislocation. Biomed. Res. Int. 2019, 2019, 7943636. [CrossRef] [PubMed]

42. Jeuken, R.M.; Vles, G.F.; Jansen, E.J.P.; Loeffen, D.; Emans, P.J. The Modified Hedgehog Technique to Repair Pure Chondral Shear-off Lesions in the Pediatric Knee. Cartilage 2019. [CrossRef] [PubMed]

43. Beckert, M.W.; Klitzman, R.G. Large chondral fragment of the lateral femoral condyle treated with arthroscopic internal fixation in an elite young athlete-A case report. Sicot. J. 2020, 6, 1. [CrossRef]

44. Ogura, T.; Sakai, H.; Asai, S.; Fukuda, H.; Takahashi, T.; Kanisawa, I.; Yamaura, I.; Tsuchiya, A.; Forney, M.; Winalski, C.S.; et al. Clinical and Radiographic Outcomes After Fixation of Chondral Fragments of the Knee in 6 Adolescents Using Autologous Bone Pegs. Orthop. J. Sports Med. 2020, 8, 2325967120963050. [CrossRef] [PubMed]

45. Rüther, H.; Seif Amir Hosseini, A.; Frosch, S.; Hoffmann, D.; Lotz, J.; Lehmann, W.; Streit, U.; Wachowski, M.M. Refixation of osteochondral fragments with resorbable polylactid implants: Long-term clincal and MRI results. Unfallchirurg 2020, 123, 797-806. [CrossRef] [PubMed]

46. Zhou, S.; Cai, M.; Huang, K. Treatment of Osteochondral Fracture of the Lateral Femoral Condyle with TWINFIX Ti Suture Anchor " $X$ "-Shaped Internal Fixation under Arthroscopy: A Surgical Technique and Three Cases Report. Orthop. Surg. 2020, 12, 679-685. [CrossRef]

47. Dhawan, A.; Hospodar, P.P. Suture fixation as a treatment for acute traumatic osteochondral lesions. Arthroscopy 1999, 15, 307-311. [CrossRef]

48. Vogel, L.A.; Fitzsimmons, K.P.; Lee Pace, J. Osteochondral Fracture Fixation with Fragment Preserving Suture Technique. Arthrosc. Tech. 2020, 9, e761-e767. [CrossRef] [PubMed]

49. Reilingh, M.L.; Murawski, C.D.; DiGiovanni, C.W.; Dahmen, J.; Ferrao, P.N.F.; Lambers, K.T.A.; Ling, J.S.; Tanaka, Y.; Kerkhoffs, G.M.M.J. Fixation Techniques: Proceedings of the International Consensus Meeting on Cartilage Repair of the Ankle. Foot Ankle Int. 2018, 39, 23S-27S. [CrossRef] [PubMed]

50. Zhou, Y.; Pan, Y.; Wang, Q.; Hou, Z.; Chen, W. Hoffa fracture of the femoral condyle: Injury mechanism, classification, diagnosis, and treatment. Medicine (Baltimore) 2019, 98, e14633. [CrossRef] [PubMed]

51. Arastu, M.H.; Kokke, M.C.; Duffy, P.J.; Korley, R.E.; Buckley, R.E. Coronal plane partial articular fractures of the distal femoral condyle: Current concepts in management. Bone Joint J. 2013, 95, 1165-1171. [CrossRef] [PubMed]

52. Say, F.; Kuyubaş, N. Arthroscopy-Assisted Fixation of an Intra-Articular Medial Femoral Condyle (Hoffa) Fracture of the Knee: A Case Report. JBJS Case Connect. 2014, 4, e109. [CrossRef] [PubMed]

53. Goel, A.; Sabat, D.; Agrawal, P. Arthroscopic-assisted fixation of Hoffa fracture: A case report and description of technique. J. Clin. Orthop Trauma 2016, 7, 61-65. [CrossRef] [PubMed]

54. Ackermann, C.; Frings, J.; Alm, L.; Frosch, K.H. Arthroscopic Controlled Closed Reduction and Percutaneous Fixation of Posterolateral Tibia Plateau Impression Fractures. Arthrosc. Tech. 2019, 8, e867-e874. [CrossRef] [PubMed]

55. Caggiari, G.; Ciurlia, E.; Ortu, S.; Sirianni, R.; Bonini, L.; Pisanu, F.; Doria, C.; Manca, M. Osteochondral avulsion fracture of the posteromedial tibial plateau. Trauma Case Rep. 2020, 25, 100281. [CrossRef] [PubMed]

56. Melugin, H.P.; Bernard, C.D.; Camp, C.L.; Stuart, M.J.; Saris, D.B.F.; Nakamura, N.; Krych, A.J. Tibial Plateau Cartilage Lesions: A Systematic Review of Techniques, Outcomes, and Complications. Cartilage 2019. [CrossRef] [PubMed]

57. Melugin, H.P.; Desai, V.S.; Levy, B.A.; Tanaka, Y.; Horibe, S.; Nakamura, N.; Krych, A.J. Osteochondritis Dissecans of the Knee: Short-Term Outcomes of a Hybrid Technique to Restore a Partially Salvageable Progeny Fragment. Cartilage 2020, 11, 300-308. [CrossRef] [PubMed]

58. Kaibara, T.; Kondo, E.; Matsuoka, M.; Iwasaki, K.; Onodera, T.; Momma, D.; Seito, N.; Mikami, S.; Iwasaki, N. Large osteochondral defect in the lateral femoral condyle reconstructed by Atelocollagen-associated autologous chondrocyte implantation combined with anterior cruciate ligament reconstruction. BMC Musculoskelet. Disord. 2020, 21, 494. [CrossRef] [PubMed]

59. Brucker, P.U.; Braun, S.; Imhoff, A.B. Mega-OATS technique-autologous osteochondral transplantation as a salvage procedure for large osteochondral defects of the femoral condyle. Oper. Orthop. Traumatol. 2008, 20, 188-198. [CrossRef] [PubMed] 
60. Kato, Y.; Chavez, J.; Yamada, S.; Hattori, S.; Takazawa, S.; Ohuchi, H. A large knee osteochondral lesion treated using a combination of osteochondral autograft transfer and second-generation autologous chondrocyte implantation: A case report. Regen Ther. 2019, 10, 10-16. [CrossRef] [PubMed]

61. Schneider, S.; Ossendorff, R.; Holz, J.; Salzmann, G.M. Arthroscopic Minced Cartilage Implantation (MCI): A Technical Note. Arthrosc. Tech. 2021, 10, e97-e101. [CrossRef] 